\title{
Design Technique of Bandpass FIR filter using Various Window Function
}

\author{
Rohit Patel $^{1}$, Er. Mukesh Kumar ${ }^{2}$, Prof. A.K. Jaiswal ${ }^{3}$, Er. Rohini Saxena ${ }^{4}$ \\ ${ }^{1} P G$ Student, ECE , SSET, SHIATS, Allahabad, India \\ ${ }^{2,4}$ Assistant Professor, ECE, SSET, SHIATS Allahabad, India \\ ${ }^{3}$ Head Of Department, ECE, SSET, SHIATS, Allahabad, India
}

\begin{abstract}
Filter is one of the most important part of communication system. Without digital filter we cannot think about proper communication because noise occurs in channel. For removing noise or cancellation of noise we use various type of digital filter. In this paper we propose design technique of bandpass FIR filter using various type of window function. Kaiser window is the best window function in FIR filter design. Using this window we can realize that FIR filter is simple and fast.
\end{abstract}

Keywords: FIR filter, LTI, bandpass filter, MATLAB

\section{Introduction}

In different areas digital filter design techniques are widely used. The digital filter consist of both software and hardware implementation. In the digital filter, the input and output signals are digital or discrete time sequence. Digital filters are linear time invariant (LTI) systems which are characterized by unit sample response. These filters are portable and highly flexible. It has minimum or negligible interference noise and other effects. In storage and maintenance digital filters are easier. Digital filters reduce the failure time. Digital filters are categorized in two parts as finite impulse response (FIR) and infinite impulse response (IIR). In comparison to IIR filters, the FIR filters have greater flexibility to control the shape of their magnitude response. According to the frequency characteristics digital filter can be divided-lowpass, highpass, bandpass, and bandstop.The realization of FIR filter is non-recursive in comparison to IIR filter. Bandpass filtering plays an important role in DSP applications. It can be used to pass the signals according to the specified frequency passband and reject the frequency other than the passband specification. Then the filtered signal can be further used for the signal feature extraction. Filtering can also be applied to perform applications such as noise reduction, frequency boosting, digital audio equalizing, and digital crossover, among others.

\subsection{Basic Concept of FIR filter}

\section{Fir Digital Filter}

The basic structure of FIR filter consists of multipliers, delay elements and adders to create the filter's output. The difference equation of $\mathrm{N}$ order of the recursive digital filters (FIR) can be represented as:

$$
\mathrm{y}(\mathrm{n})=\sum_{k=0}^{N-1} h(n) x(n-k)=\sum_{\mathrm{k}=0}^{\mathrm{N}-1} b_{k} x(n-k)
$$

Where, $y(n)$ is the output signal, $h(n)$ is the filter coefficients and $\mathrm{k}$ is the order of the filters.

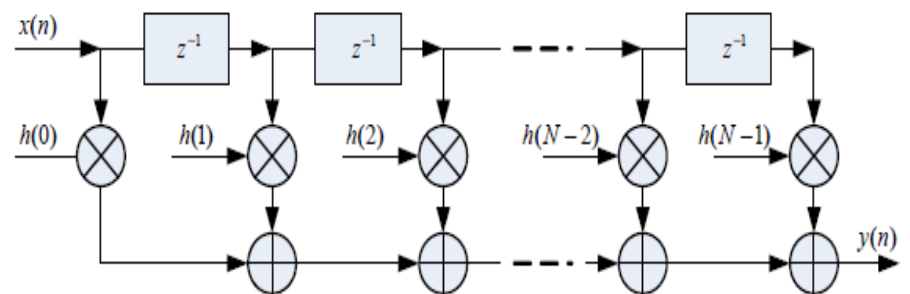

Figure.1 N-order FIR digital filter block diagram

We can express the output signal in frequency domain by convolution of the input signal $x(n)$ and the impulse response $\mathrm{h}(\mathrm{n})$.

$$
\mathrm{Y}(\mathrm{n})=\mathrm{x}(\mathrm{n}) * \mathrm{~h}(\mathrm{n})
$$

The output signal is determined as, 


$$
\mathrm{Y}(\mathrm{n})=\mathrm{x}(0) * \mathrm{~h}(\mathrm{n})+\mathrm{x}(1) * \mathrm{~h}(\mathrm{n}-1)+\mathrm{x}(2) * \mathrm{~h}(\mathrm{n}-2)+\ldots \ldots . . \mathrm{x}(\mathrm{n}) \mathrm{h}(0)
$$

In differential equation, the coefficient $b_{k}$ equals to the successive value $\mathrm{h}(\mathrm{n})$ of unit-sample response. The system function $\mathrm{H}(\mathrm{z})$ can be expressed as:

$$
\mathrm{H}(\mathrm{z})=\sum_{k=0}^{N-1} b_{k} z^{-k}
$$

$\mathrm{H}(\mathrm{z})$ is polynomial of $z^{-1}$. This means that all poles are only plotted at the origin of the Z-plane.

FIR filters can be designed in different ways, for example window method, frequency sampling method, weighted least squares method, minimax method and equiripple method. Out of these methods, the window technique is most conventional method for designing FIR filters.

\subsection{Window function method of FIR filter design}

The basic design principles of window function are to calculate $h_{d}(\mathrm{n})$ by the anti-Fourier transform based on the ideal demanded filter frequency response $H_{d}\left(e^{j \omega}\right)$. The formula of $h_{d}(\mathrm{n})$ is shows as

$$
h_{d}(\mathrm{n})=\frac{1}{2 \pi} \int_{-\pi}^{\pi} H_{d}\left(e^{j \omega}\right)
$$

Because $h_{d}(\mathrm{n})$ is infinitely long, we have to deal with it by window function to get to the unit impulse response $\mathrm{h}(\mathrm{n})$. Now it is written as

$$
\mathrm{h}(\mathrm{n})=\mathrm{w}(\mathrm{n}) \cdot h_{d}(\mathrm{n})
$$

Where $\mathrm{w}(\mathrm{n})$ is the window function. Fixed window and adjustable window are the two categories of window function. Blackman window, Hanning, Hamming and rectangular window are mostly used fixed window function. Kaiser window is a type of adjustable window function.

\subsubsection{Hanning window}

The Hanning window is a raised cosine window and can be used to reduce the side lobes while preserving a good frequency resolution compared to the rectangular window. The hanning window is defined as

\subsubsection{Hamming window}

$$
w(n)=\left\{\begin{array}{cl}
0.5\left(1-\cos \frac{2 \pi n}{M-1}\right), & \text { for } n=0 \text { to } N-1 \\
0, & \text { elsewhere }
\end{array}\right.
$$

The hamming window is, like the Hanning window, also a raised cosine window. The hamming window exhibits similar characteristics to the Hanning window but further suppress the first side lobe. The hamming window is defined as

$$
w(n)=\left\{\begin{array}{cl}
\left(0.54-0.46 \cos \left(\frac{2 \pi n}{N}\right)\right), & \text { for } n=0 \text { to } N-1 \\
0, & \text { elsewhere }
\end{array}\right.
$$

\subsubsection{Blackman window}

The Blackman window is similar to Hanning and Hamming windows. An advantage with Blackman window over other windows is that it has better stop band attenuation and with less pass band ripple. The Blackman window is defined as

$$
w(n)=\left\{\begin{array}{cc}
\left(0.42-0.5 \cos \left(\frac{2 \pi n}{N}\right)\right)+0.08 \cos \left(\frac{4 \pi n}{N}\right), & \text { for } n=0 \text { to } N-1 \\
0, & \text { elsewhere }
\end{array}\right.
$$

\subsubsection{Kaiser window}

The Kaiser window with parameter $\beta$ is defined as

$$
\mathrm{w}(\mathrm{n})= \begin{cases}\frac{\mathrm{I}_{0}\left(\beta \sqrt{1-(2(\mathrm{n}+1) /(\mathrm{N}+1))^{2}}\right.}{\mathrm{I}_{0}}, & \text { for } n-0 \text { to } \mathrm{N}-1 \\ 0, & \text { elsewhere }\end{cases}
$$


The parameter $\beta$ determines the shape of the window and thus controls the trade-off between main-lobe width and side-lobe amplitude.

\section{Fir Filter Design Using Fda Tool}

The Filter Design and Analysis (FDA) tool works with MATLAB and the signal processing toolbox to provide a complete environment for start to finish filter design. The FDA tool supports many advanced techniques not available in SP tool. FDA tool is used to - design filters, quantize filter, analyze filter, modify existing filter designs, realize simulink models of quantized direct form FIR filters.

\subsection{Filter Specifications}

Table.1 Filter specification

\begin{tabular}{|l|l|}
\hline Parameters & Values \\
\hline Filter Type & Bandpass \\
\hline Design method & $\begin{array}{l}\text { FIR window }(\beta=3.4 \text { for Kaiser } \\
\text { window only) }\end{array}$ \\
\hline Filter order & 38,48 \\
\hline Sampling frequency & 100 \\
\hline Lower cut-off frequency & 10 \\
\hline Upper cut - off frequency & 20 \\
\hline
\end{tabular}

In FDA toolbox of MATLAB ,From the table.1, first choose "Bandpass" in option of "Filter Type", choose "FIR Window " in option of "Design Method", choose " Blackman, hamming, hanning and Kaiser " one by one in option of "Window ", set "Beta" = 3.4 only for kaiser window. Assign "Specify order"=38 and 48 one by one in options of "Filter Order" ; Sampling frequency Fs $=100 \mathrm{~Hz}$,Since use the method of window function to design ,only provide band pass lower cutoff frequency $\mathrm{fc} 1=10 \mathrm{~Hz}$ and band pass cap cut-off frequency fc2 $=20 \mathrm{~Hz}$. After setting, click "Design Filter", will obtain the FIR filter by design. Through the menu options "Analysis", amplitude frequency response and phase frequency response, zero-pole assignment, coefficient of filter and various characteristics of filter can be showed up.l

\subsection{Blackman Window}

\section{Result And Simulation}

From table 1 we analyzed the filter using Blackman window by FDA tool in the MATLAB and the response of the filter is given in figure 2 and 3 respectively at the order 38 and 48 .

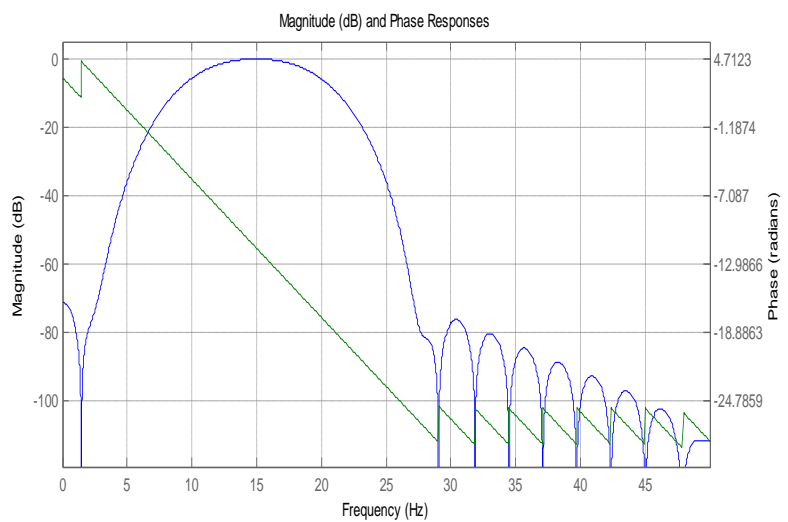

Figure.2 FIR Blackman window $(\mathrm{N}=38)$

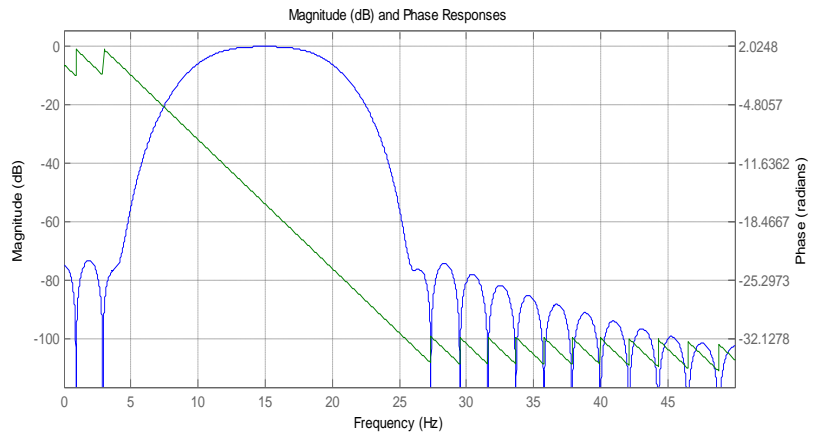

Figure.3 FIR Blackman window $(\mathrm{N}=48)$ 


\subsection{Hamming Window}

We analyzed the filter using Hamming window or fixed widow by FDA tool in the MATLAB and the response of the filter is given in figure 4 and 5 respectively at the order 38 and 48.

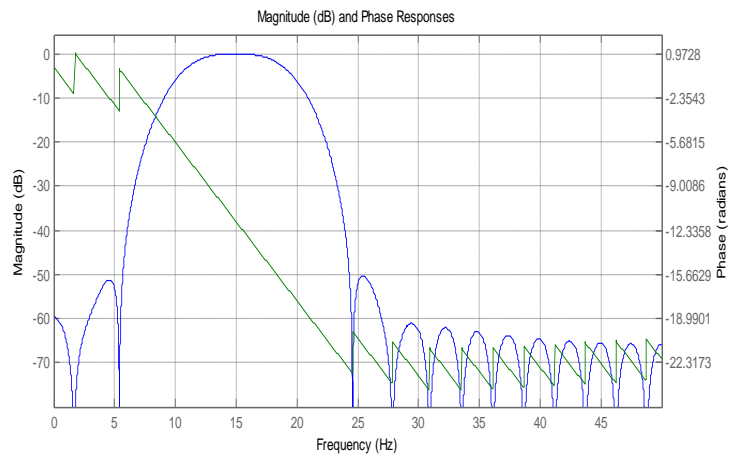

Figure.4 FIR Hamming window (N=38)

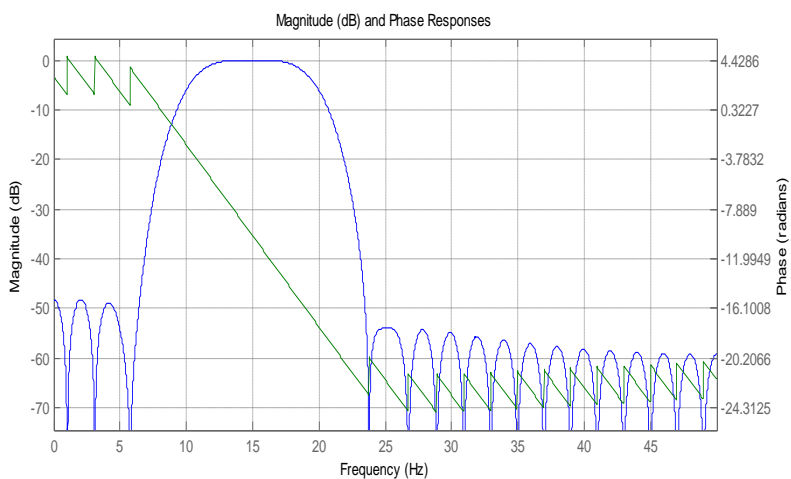

Figure.5 FIR Hamming window $(\mathrm{N}=48)$

\subsection{Hanning Window}

We analyzed the filter using Hanning window or fixed widow by FDA tool in the MATLAB and the response of the filter is given in figure 4 and 5 respectively at the order 38 and 48 .

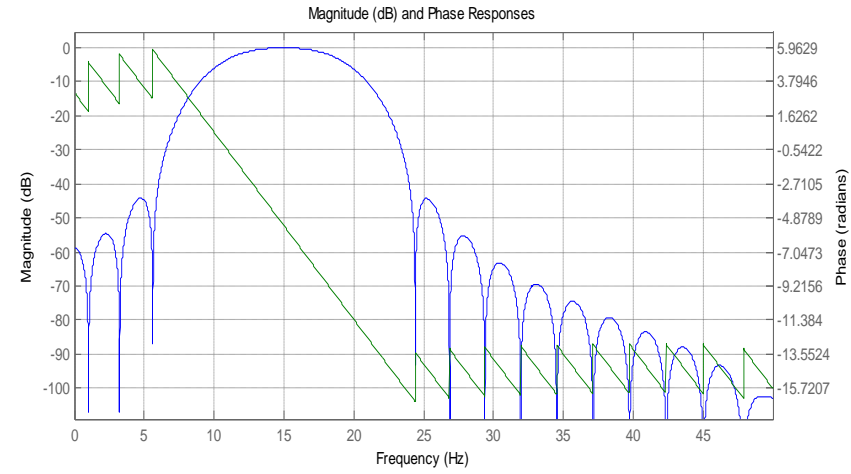

Figure.6 FIR Hanning window $(\mathrm{N}=38)$

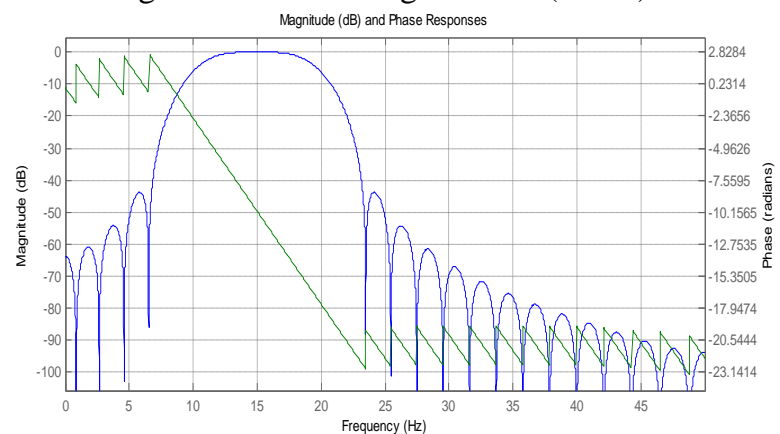

Figure.7 FIR Hanning window $(\mathrm{N}=48)$ 


\subsection{Kaiser Window}

We analyzed the filter using Kaiser window by FDA tool in the MATLAB and the response of the filter is given in figure 8 and 9 respectively at the order 38 and 48 .

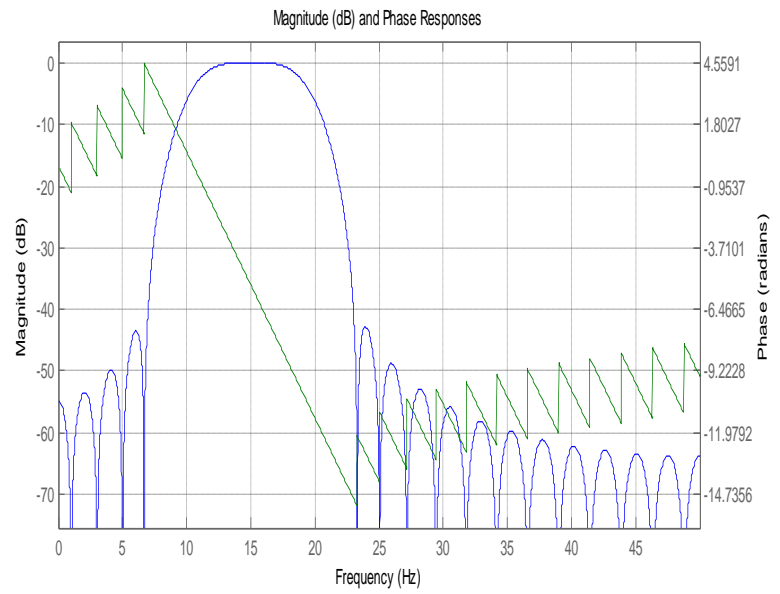

Figure.8 FIR Kaiser window ( $\mathrm{N}=38)$

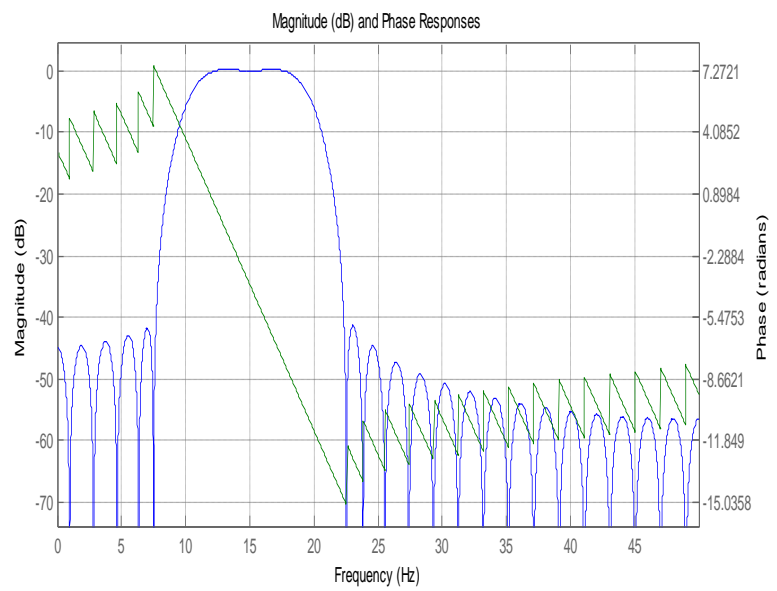

Figure.9 FIR Kaiser window (N=48)

Table.2 Comparison between different window techniques

\begin{tabular}{|c|c|c|c|}
\hline $\begin{array}{l}\text { Window } \\
\text { technique }\end{array}$ & $\begin{array}{l}\text { Order of the } \\
\text { filter }\end{array}$ & $\begin{array}{l}\text { Width of main } \\
\text { lobe }\end{array}$ & No. of side lobes \\
\hline \multirow{2}{*}{$\begin{array}{l}\text { Blackman } \\
\text { Window }\end{array}$} & 38 & 0.085938 & 9 \\
\hline & 48 & 0.066406 & 13 \\
\hline \multirow{2}{*}{$\begin{array}{l}\text { Hamming } \\
\text { Window }\end{array}$} & 38 & 0.066406 & 12 \\
\hline & 48 & 0.054688 & 16 \\
\hline \multirow{2}{*}{$\begin{array}{l}\text { Hanning } \\
\text { Window }\end{array}$} & 38 & 0.074219 & 13 \\
\hline & 48 & 0.058594 & 17 \\
\hline \multirow{2}{*}{$\begin{array}{l}\text { Kaiser }(\beta=3.4) \\
\text { Window }\end{array}$} & 38 & 0.058594 & 16 \\
\hline & 48 & 0.046875 & 20 \\
\hline
\end{tabular}

From the table 2 we can see that as the order of the FIR filter increases the number of the side lobes also increases and width of the main lobe is decreased, that it is tending to sharp cut off that is the width of the main lobe decreased. If the width of the main lobe reduces then the number of the side lobes gets increased. So there should be a compromise between attenuation of side lobes and width of main lobe. On comparing all methods, the Blackman has the smallest side lobes at any order but the width of the main lobe is increased. In the Kaiser window for the lower order the width of the major lobe is less than the other windows. The Kaiser window gives best result. Therefore it is most commonly used window for FIR filter design.

\section{Conclusion}

Digital filter can play a major role in speech signal processing applications such as, speech filtering, speech enhancement, noise reduction and automatic speech recognition. The kaiser window gives the minimum 
mainlobe width 0.046875 for filter order 48 which means this window has less transition width and introduces more ripple.

\section{References}

[1] S. Salivahanan, A. Vallavaraj, C. Gnanaapriya, "Digital Signal Processing”, Tata McGraw-Hill, 2000.

[2] Sonika Gupta, Aman Panghal Performance, "Performance Analysis of FIR Filter Design by Using Rectangular, Hanning and Hamming Windows Methods", International Journal of Advanced Research in Computer Science and Software Engineering Volume 2, Issue 6, June 2012.

[3] Chonghua Li, "Design and Realization of FIR Digital Filters Based on MATLAB”, ,IEEE 2010.

[4] Saurabh Singh Rajput, Dr. S. S. Bhadauria, "Implementation of FIR Filter using Efficient Window Function and its Application in Filtering a Speech Signal”, International Journal of Electrical, Electronics and Mechanical Controls Volume 1, Issue 1, November 2012 . 